\title{
Energy Efficient Proportionally Fair Uplink Offloading for IP Flow Mobility
}

\author{
V. Miliotis ${ }^{\dagger}$, L. Alonso ${ }^{\dagger}$ and C. Verikoukis ${ }^{\star}$ \\ ${ }^{\dagger}$ Technical University of Catalonia, Department of Signal Theory and Communications, Barcelona, Spain \\ ${ }^{\star}$ Telecommunications Technological Centre of Catalonia (CTTC), Castelldefels, Spain \\ Email: \{vasileios.miliotis, luisg\}@tsc.upc.edu, cveri@cttc.es
}

\begin{abstract}
The continuous increase of mobile data demand has led to mobile data offloading as a solution for the network congestion problem. The majority of the state-of-the-art is focused on the downlink offloading, while the change of mobile user habits, like mobile content creation and uploading, makes uplink offloading a rising issue. In this work we focus on the uplink offloading using Ip Flow Mobility (IFOM). IFOM allows a LTE mobile User Equipment (UE) to maintain two concurrent data streams, one through LTE and the other through WiFi access technology, that presents uplink limitations due to the inherent fairness design of IEEE 802.11 DCF. In this paper, we propose a weighted Proportionally Fair Bandwidth (PFB) allocation algorithm, for the WiFi access, in conjunction with a pricing-based rate allocation for the LTE uplink, aiming to improve the energy efficiency of the UEs that operate under the concurrent use of access technologies that IFOM provides. We present a theoretical analysis of the proposed schemes and evaluate their performance in terms of energy efficiency through simulations.
\end{abstract}

Keywords-Offloading, IFOM, Proportional Fairness, Linear Pricing.

\section{INTRODUCTION}

The continuous increase of cellular data demand, that is already witnessed, is the main driving force for cellular network operators towards the capital investments on upgrades of their cellular network infrastructures into $4 \mathrm{G}$ systems, as LTE. With the upgrade of their networks, cellular network providers aim to be able to serve the requested traffic by their customers. Despite the upgrade of the cellular infrastructures, the pace of the increase of the data traffic demand [1] puts pressure to the cellular network providers, as traffic congestion is not avoided. These facts have led the research community to propose offloading techniques that will leverage the mitigation of the overload of the cellular network spectrum and the network's traffic congestion. According to the work of Paul et al. [2] on the dynamics of cellular data networks, downloads dominate uploads with more than $75 \%$ of the traffic coming from download traffic. On the other hand, smartphone applications slowly change the users attitude, transforming them into content creators. Facebook, Twitter, Youtube and Instagram are some of the main applications that let users upload their content (videos, photos, audio, text and combinations of them) at the time of creation. This change of use habits is highly demanding in terms of energy consumption, as in LTE, uploading is nearly eight times more energy consuming compared to downloading [3]. Considering the solution of offloading the uplink traffic of users that are in the range of WiFi APs, the battery life of mobile users will be extended and at the same time the uplink load of an eNodeB will be mitigated.
With the release-10 of 3GPP, a UE in LTE networks is able to concurrently maintain connections with the cellular network and a WiFi AP, in order to offload part of its traffic. The scheme that allows this connectivity is named IP Flow Mobility (IFOM) [4]. IP Flow Mobility is currently being standardized by 3GPP [5]. This technology allows an operator or a UE to shift an IP flow to a different radio access technology, without disrupting any ongoing communication. Consider a UE connected to a cellular base station having multiple simultaneous flows. For example, it maintains a voice call and a file upload, and it is moving into the range of a $\mathrm{WiFi}$ AP. The UE may shift the file upload on the WiFi network and when it moves out of the AP coverage it will make a seamless shift of the flow back to the cellular network. Another example is the division of a UE's data flow into two sub-flows and the service of each sub-flow by different radio access technologies, as proposed in [6]. Scheduling algorithms for this approach are presented in [7]. A question that arises from the IFOM uplink offloading scheme is how the UEs will offload part of their data through WiFi with fairness, and how the rest of the data will be uploaded through LTE. For the LTE uplink rate allocation we propose a two-stage pricing algorithm. We follow a linear pricing scheme, that was used in [8] and [9].

The rest of the paper is organised as follows. In Section II we present the system model and we analyse the energy consumption of the LTE and WiFi network interface cards of UEs. In Section III we present the PFB algorithm and in Section IV we analyse the proposed LTE pricing scheme for linear pricing. Section V contains the simulation results and Section VI concludes our work.

\section{SySTEM MODEL}

We consider a LTE macro-cell and we focus on its coverage area that is also partially covered by several WiFi APs that belong to the same LTE provider. All UEs are equipped with a WiFi network interface card in addition to their LTE connectivity. We assume that $N$ LTE UEs are concurrently under the coverage of the macro-cell and one of the deployed APs, and they need to upload a file (e.g. a photo or a video) through a mobile application. The used applications are assumed to be able to divide an IP flow into two sub-flows and to define the size of each one. The UEs are able to use concurrently the two access technologies with IFOM and direct one sub-flow to LTE and the other to WiFi. The data volume of the upload need of $\mathrm{UE}_{i}$ is equal to $K_{i}$ bits, where $i=(1, \ldots, N)$. The data needs $K_{i}$ are a priori known to the WiFi AP. The AP has a high bandwidth backbone (e.g. fiber connection). Thus, the 
bottleneck of this route lies in the wireless uplink access of the WiFi connection. The described scheme is applied to each one of the WiFi APs and we investigate the uplink data offloading for a time horizon equal to $\Delta T$. During this time horizon, we assume that the channel characteristics between each $\mathrm{UE}_{i}$ and the LTE macro-cell are described by a normalized spectrum efficiency $\theta_{i} \in[0,1]$. Thus, after the bandwidth allocation, $\mathrm{UE}_{i}$ is allocated an uplink rate equal to $R_{i}^{L T E}$, but the actual achieved uplink rate is equal to $\theta_{i} R_{i}^{L T E}$.

\section{A. LTE Uplink Power Model}

Regarding the LTE uplink power level of the UE, we adopt the energy model proposed by Huang et al. in [3]. According to this model the power level of the $\mathrm{UE}_{i}$ 's LTE interface during uplink transmission is expressed as

$$
P_{i}^{L T E}=\alpha_{u} R_{i}^{L T E}+\beta[\mathrm{mW}]
$$

where $\alpha_{u}$ is the uplink transmission power per Mbps, $R_{i}^{L T E}$ is the LTE uplink rate of $\mathrm{UE}_{i}$ (in Mbps) and $\beta$ is the base power of the LTE card.

\section{B. IEEE 802.11 DCF Energy Consumption in the Uplink}

The uplink access mechanism of IEEE 802.11 DCF [10] is based on contention among users that are willing to transmit data to the AP and try to avoid collisions following the standard's binary exponential back-off algorithm. Following Bianchi's analysis [11] for saturated traffic conditions we notice that the throughput of a user that tries to upload data through WiFi is significantly affected by the number of users that are under the coverage of the same AP. The per user throughput $S(N)$ (in Mbps), where $N$ is the number of contending users, is expressed as

$$
S(N)=\frac{P_{s}(N) P_{t r}(N) E[P]}{N\left[\left(1-P_{t r}(N)\right) \sigma+P_{t r}(N) P_{s}(N) T_{s}+P_{t r}(N)\left(1-P_{s}(N) T_{c}\right)\right]}
$$

$E[P], T_{s}, T_{c}$ and $\sigma$ correspond to the average payload of a packet, the duration of a successful transmission, the duration of a collision and the time slot's duration respectively. $P_{t r}(N)$ is the probability that there is at least one transmission in a considered time slot and $P_{s}(N)$ is the probability that an occurring transmission is successful. A user's energy efficiency $E E(N)$ (in bits/Joule), as a function of the number of contending users $N$ is expressed as

$E E(N)=\frac{P_{s}(N) P_{t r}(N) E[P]}{N\left[\left(1-P_{t r}(N)\right) E_{i}+P_{t r}(N) P_{s}(N) E_{s}+P_{t r}(N)\left(1-P_{s}(N) \underset{(3)}{\left.E_{c}\right)}\right)\right.}$

where $E_{i}, E_{s}$ and $E_{c}$ correspond to the energy consumption of a user during an idle, a successful transmission and a collision period. The duration of a successful transmission is equal to $T_{s}=T_{H}+T_{E[P]}+T_{S I F S}+T_{A C K}+T_{D I F S}$. The duration of a collision period is equal to $T_{c}=T_{H}+T_{E[P]}+T_{D I F S}$, and the duration of an idle period is equal to a time slot $\sigma$. Where $T_{H}$ is the transmission duration of the PHY and MAC headers and $T_{E[P]}$ the average transmission duration of a packet's payload for transmission rate equal to $R^{W i F i}=54 \mathrm{Mbps}$. Taking these duration expressions into consideration we analytically express the energy consumption values of (3) in (4).

$$
\begin{aligned}
& E_{s}=P_{T x}\left(T_{H}+T_{E[P]}\right)+P_{i d l e}\left(T_{S I F S}+T_{D I F S}\right)+P_{R x} T_{A C K} \\
& E_{c}=P_{T x}\left(T_{H}+T_{E[P]}\right)+P_{i d l e} T_{D I F S} \\
& E_{i}=\sigma P_{i d l e}
\end{aligned}
$$

where $P_{i d l e}, P_{T x}$ and $P_{R x}$ are the power levels of the user's 802.11 network interface card.

\section{Uplink Offloading Energy Consumption}

Every UE under the concurrent coverage of the two access technologies will have the opportunity to offload $w_{i} K_{i}$ bits through the WiFi AP, where $w_{i} \in[0,1]$ for $i=(1, \ldots, N)$. The remainder data volume $\left(1-w_{i}\right) K_{i}$ is transmitted through the LTE connection of each UE. Every $\mathrm{UE}_{i}$ with data needs equal to $K_{i}$ that offloads its uplink according to $w_{i}$ will present energy consumption $E C_{i}(N)$, which is expressed as

$$
E C_{i}(N)=\left(1-w_{i}\right) K_{i} \frac{P_{i}^{L T E}}{\theta_{i} R_{i}^{L T E}}+w_{i} K_{i} \frac{1}{E E(N)} \text { [Joule] }
$$

For the WiFi uplink offloading we provide a weighted proportionally fair allocation algorithm over the data needs and the LTE channel conditions of the UEs. For the LTE uplink of the $\left(1-w_{i}\right) K_{i}$ data volume of each $\mathrm{UE}_{i}$ we provide a two stage pricing algorithm for the LTE uplink rate allocation. Based on these two parts of the IFOM uplink offloading we are able to calculate each $\mathrm{UE}_{i}$ 's energy consumption according to (5).

\section{Weighted Proportionally FAir WiFi ACCESS}

The UEs offload part of their data needs through the WiFi according to the Proportionally Fair Bandwidth (PFB) allocation algorithm that we propose. Each $\mathrm{UE}_{i}$ is allocated bandwidth equal to $r_{i}, i=(1, \ldots, N)$, such as $\sum_{i=1}^{N} r_{i} \leq R_{i}^{W i F i}$. The allocation is proportionally fair over the ratio $\rho_{i}=K_{i} / \theta_{i}$. According to the definition of proportional fairness by Kelly et al. [12], a vector of rate allocation $\mathbf{r}=\left(r_{1}, \ldots, r_{N}\right)$ is proportionally fair if it is feasible, that is $\mathbf{r} \geq 0$ and $\sum_{i=1}^{N} r_{i} \leq R_{i}^{W i F i}$ and if for any other feasible vector $\mathbf{r}^{*}$, regarding the proportional fairness over the ratio $\rho_{i}$ of each $\mathrm{UE}_{i}$, the aggregate of proportional changes is zero or negative and is expressed as

$$
\sum_{i=1}^{N} \rho_{i} \frac{r_{i}^{*}-r_{i}}{r_{i}} \leq 0
$$

which can be rewritten as

$$
\sum_{i=1}^{N} \rho_{i}\left(\log \left(r_{i}\right)\right)^{\prime} d r_{i} \leq 0
$$

It follows from (7) that the proportionally fair allocation solution represents a maximum of the utility function $U_{i}(\mathbf{r})=$ $\sum_{i=1}^{N} \rho_{i} \log \left(r_{i}\right)$. Consequently, in order to find the proportionally fair solution we have to solve the maximization problem 
described as follows

$$
\begin{array}{ll}
\max _{\mathbf{r}} & \sum_{i=1}^{N} \rho_{i} \log \left(r_{i}\right) \\
\text { subject to } & \sum_{i=1}^{N} r_{i} \leq R^{W i F i} \\
\text { and } & r_{i} \geq 0, \forall i=1, \ldots N
\end{array}
$$

The problem has a unique solution since the objective function is strictly concave and the constraint set is convex. To solve this problem, we relax the constraints and define the Lagrangian [13], changing $r_{i} \geq 0$ to $-r_{i} \leq 0$

$L(\mathbf{r}, \mu)=\sum_{i=1}^{N} \rho_{i}\left(\log \left(r_{i}\right)\right)-\mu_{0}\left(\sum_{i=1}^{N} r_{i}-R^{W i F i}\right)+\sum_{i=1}^{N} \mu_{i} r_{i}$

where $\mu_{0} \geq 0$ and $\mu_{i} \geq 0, i=(1, \ldots, N)$. Following, we take the Karush-Kuhn-Tucker (KKT) optimality conditions. Starting with the stationarity condition we have

$$
\nabla_{r_{i}} L(\mathbf{r}, \mu)=\frac{\rho_{i}}{r_{i}}-\mu_{0}+\mu_{i}=0
$$

since $\rho_{i}>0$, then $\mu_{0}>\mu_{i}$, which also means $\mu_{0}>0$. From the complementary slackness conditions we have

$$
\begin{gathered}
\mu_{0}\left(R^{W i F i}-\sum_{i=1}^{N} r_{i}\right)=0 \\
\mu_{i} r_{i}=0 \\
\mu_{0} \geq 0 \text { and } \mu_{i} \geq 0, i=(1, \ldots, N)
\end{gathered}
$$

and since $\mu_{0}>0$, we know that

$$
\sum_{i=1}^{N} r_{i}=R^{W i F i}
$$

which means that $r_{i}, i=(1, \ldots, N)$, cannot be zero. Thus, forcing $\mu_{i}=0, \forall i=(1, \ldots, N)$ we have from (10)

$$
r_{i}=\frac{\rho_{i}}{\mu_{0}}
$$

Combining (14)) and (15) we have the optimal solution which represents the weighted proportionally fair solution

$$
r_{i}=\frac{\rho_{i}}{\sum_{i=1}^{N} \rho_{i}} R^{W i F i}
$$

\section{A. Implementation Consideration}

In the PFB algorithm we aim to allocate exclusive access periods to each $\mathrm{UE}_{i}$ equal to $t_{i}$, for $i=(1, \ldots, N)$. In these periods the UEs will be able to transmit through the WiFi AP with throughput $R^{W i F i}=S(1)$. We transform the proportionally fair bandwidth allocation into proportionally fair airtime allocation by having $r_{i} \Delta T=t_{i} S(1)$. Now, the weighted proportionally fair airtime allocation is equal to

$$
t_{i}=\frac{\rho_{i}}{\sum_{i=1}^{N} \rho_{i}} \Delta T
$$

Regarding the implementation of the PFB algorithm we aim to

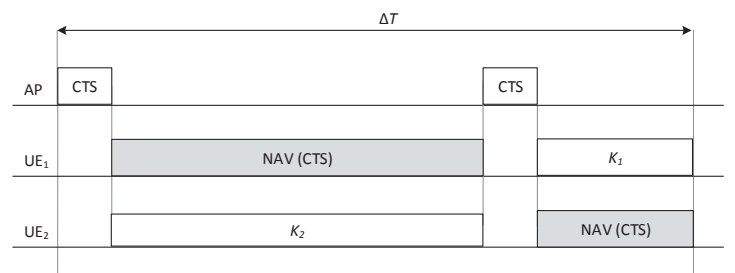

Fig. 1. An example of the PFB algorithm for two UEs.

give exclusive access to the WiFi AP to each $\mathrm{UE}_{i}$ for a period equal to $t_{i}$. To achieve that, we adopt the idea of unsolicited Clear To Send (CTS) frames initiated by the AP that was proposed in [14]. With a CTS frame the AP protects a specific UE to upload its data through WiFi, while all other UEs put their 802.11 network interface cards into sleep mode for a duration equal to the NAV information of the CTS. A timeline example for the WiFi access of the PFB algorithm for two UEs is presented in Fig. 1. We notice that due to non optimally scheduled user access, $\mathrm{UE}_{2}$ is obliged to wait for a longer period in comparison to its own access time. Even though during this waiting period $\mathrm{UE}_{2}$ 's $\mathrm{WiFi}$ card is in sleep mode, it consumes energy. We can further improve our algorithm by applying the optimal scheduling for one machine and nonpreemptive jobs which is a shortest-job-first fashion approach.

\section{B. Energy Efficiency of $P F B$}

The average per UE energy consumption of the WiFi network interface card, during the uploading phase, is expressed as

$$
E C_{T x}^{W i F i}=\frac{1}{N}\left(\sum_{i=1}^{N} \frac{\rho_{i}}{\sum_{i=1}^{N} \rho_{i}} \Delta T \frac{S(1)}{E E(1)}\right) \text { [Joule] }
$$

After scheduling the exclusive time periods $t_{i}$ in augmenting order of duration, the average per UE energy consumption of the WiFi network interface card while in sleep mode with power level $P_{\text {sleep }}^{W i F i}$, is expressed as

$$
E C_{\text {sleep }}^{W i F i}=\frac{1}{N} \sum_{i=1}^{N-1}(N-i) t_{i} P_{\text {sleep }}^{W i F i}[\text { Joule }]
$$

The average per UE energy consumption of the LTE network interface card is equal to

$$
E C^{L T E}=\frac{1}{N} \sum_{i=1}^{N}\left(\left(K_{i}-t_{i} S(1)\right) \frac{P_{i}^{L T E}}{\theta_{i} R_{i}^{L T E}}\right) \text { [Joule] }
$$

Combining (18)-(20) the average per UE energy efficiency of IFOM offloading under the PFB algorithm is expressed in (21).

$$
E_{e f f}^{P F B}=\frac{\sum_{i=1}^{N} K_{i}}{N\left(E C_{T x}^{W i F i}+E C_{\text {sleep }}^{W i F i}+E C^{L T E}\right)} \text { [bits/Joule] }
$$

\section{LTE PRICING SCHEME}

The LTE uplink power of each $\mathrm{UE}_{i}$, following the power model of (1), is a function of its LTE uplink transmission rate, $R_{i}^{L T E}$. Hereunder, we propose a two-stage LTE pricing 
scheme, where the LTE operator decides the price $p$ per unit of transmit rate $R_{i}^{L T E}$ in the first step and in the second step the UEs decide the rate for which they intend to pay as a function of the price and the spectrum efficiency they experience. We approach the pricing problem using backward induction, examining first the UEs demands (Stage II) and then the operator's decision on the price (Stage I).

\section{A. LTE Uplink Rate With Linear Pricing}

Stage II: The payoff function of the $\mathrm{UE}_{i}$, for acquiring $R_{i}^{L T E}$ quantity of uplink rate with a price $p$ per unit of rate, following the linear pricing model, is expressed as

$$
U_{i}^{l i n}\left(R_{i}^{L T E}\right)=\ln \left(1+\theta_{i} R_{i}^{L T E}\right)-p R_{i}^{L T E}
$$

This payoff function of a $\mathrm{UE}_{i}$, with normalized spectrum efficiency $\theta_{i}$, is equal to the logarithmic utility function, that expresses the diminishing return of getting additional resources, minus the linear price that the $\mathrm{UE}_{i}$ has to pay for acquiring $R_{i}^{L T E}$ quantity of rate. We notice that $U_{i}^{l i n}\left(R_{i}^{L T E}\right)$ is a concave function, since $U_{i}^{l i n}\left(R_{i}^{L T E}\right)^{\prime \prime}=$ $-\left(\theta_{i} /\left(1+\theta_{i} R_{i}^{L T E}\right)\right)^{2}<0$. Thus, it has only one maximum, and therefore the local maximum is also the global maximum. Differentiating (22) we have

$$
\frac{\partial U_{i}^{l i n}}{\partial R_{i}^{L T E}}=\frac{\theta_{i}}{1+\theta_{i} R_{i}^{L T E}}-p=0
$$

The optimal value of rate that maximizes $\mathrm{UE}_{i}$ 's payoff is

$$
R_{i}^{* L T E}= \begin{cases}\frac{1}{p}-\frac{1}{\theta_{i}}, & \text { if } p \leq \theta_{i} \\ 0, & \text { otherwise }\end{cases}
$$

Stage I: We take into consideration that the $N$ UEs that are under the coverage of the same WiFi AP are close enough to present similar channel statistics of their LTE connections. Thus, we assume that their spectrum efficiency is such that $\max \left(\theta_{i}\right)-\min \left(\theta_{i}\right)<\epsilon$, where $\epsilon>0$. Under this assumption, the operator's choice of price $p$ is such, that the UE with the $\max \left(\theta_{i}\right)$ is allocated the maximum value of the LTE uplink rate $R_{\max }^{L T E}$. The price is formed according to (25).

$$
p=\frac{\max \left(\theta_{i}\right)}{1+\max \left(\theta_{i}\right) R_{\max }^{L T E}}
$$

The provider aims to give to every $\mathrm{UE}_{i}$ the opportunity to transmit through the LTE. This means that even for the UE with the $\min \left(\theta_{i}\right)$, the quantity $1 / p-1 / \min \left(\theta_{i}\right)$ is positive. Using (25) we find the range of values of $\epsilon$ under which this rate allocation is feasible. This range is expressed as

$$
0<\epsilon \leq \max \left(\theta_{i}\right) \min \left(\theta_{i}\right) R_{\max }^{L T E}
$$

TABLE I. Simulation PARAMETERS

\begin{tabular}{ll}
\hline Parameter & Value \\
\hline LTE uplink rate (max) $R_{\max }^{L T E}$ & $5 \mathrm{Mbps}$ \\
LTE uplink power per Mbps $\alpha_{u}$ & $438.39 \mathrm{~mW} / \mathrm{Mbps}$ \\
LTE base power $\beta$ & $1288.04 \mathrm{~mW}$ \\
LTE uplink power & $\alpha_{u} R_{i}^{L T E}+\beta \mathrm{mW}$ \\
WiFi packet payload & $1500 \mathrm{bytes}$ \\
WiFi Data/ Ctrl. transmission rate & $54 / 6 \mathrm{Mbps}$ \\
WiFi Tx/ Rx/ Idle/ Sleep Power & $1900 / 1340 / 1340 / 75 \mathrm{~mW}$ \\
SIFS/ DIFS & $10 / 50 \mu \mathrm{sec}$ \\
Offloading period $\Delta T$ & $5 \mathrm{sec}$ \\
Number of UEs & $4-20$ \\
Uplink data volume per UE & $5-15 \mathrm{MB}$ \\
\hline
\end{tabular}

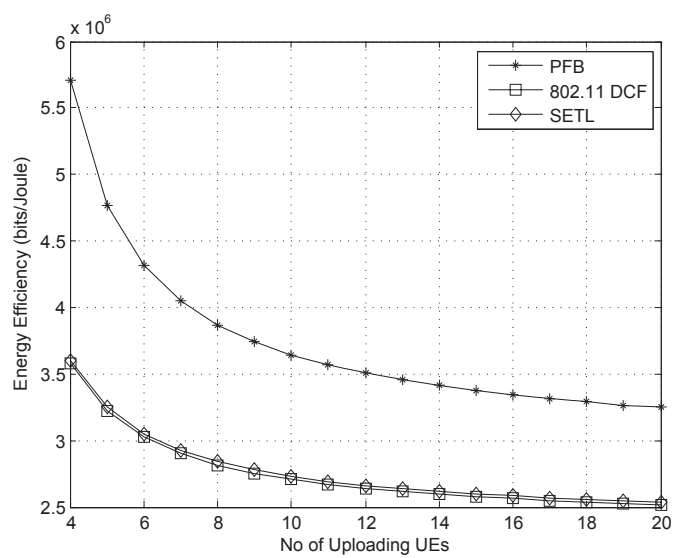

Fig. 2. Energy efficiency for $\theta_{i} \in[0.8,1]$ with linear pricing.

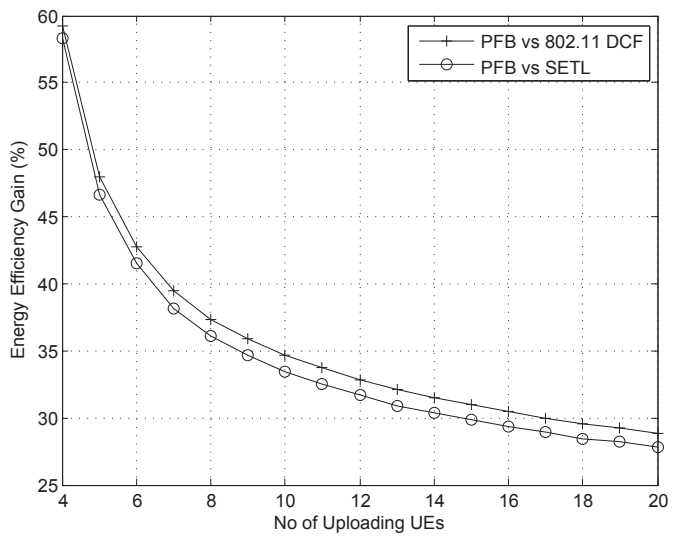

Fig. 3. Energy efficiency Gain for $\theta_{i} \in[0.8,1]$ with linear pricing.

The allocated rate to each $\mathrm{UE}_{i}$ following the linear pricing model is expressed as

$$
R_{i}^{L T E}=\frac{1+\max \left(\theta_{i}\right) R_{\max }^{L T E}}{\max \left(\theta_{i}\right)}-\frac{1}{\theta_{i}}
$$

\section{Evaluation And Simulation REsults}

We evaluate our proposed offloading schemes by running extensive simulations using MATLAB ${ }^{\mathrm{TM}}$. We run the PFB algorithm for a diverse number of UEs under the concurrent coverage of an eNodeB and a WiFi AP, namely for four to 20 UEs. We compare the performance of PFB in terms of energy efficiency with the standard 802.11 DCF and with an access mechanism titled Smart Exponential-Threshold-Linear (SETL) that was proposed in [15]. In the backoff algorithm proposed in SETL the Contention Window $(C W)$ of a 802.11 user is increasing exponentially up to a threshold that is equal to $C W_{t h}=\left(C W_{\max } / 2+C W_{\min }\right)$. After this threshold, it is increasing linearly up to $C W_{\max }$ according to $C W_{t h}+k C W_{\min }$, where $k$ is a positive integer. Regarding the LTE part of IFOM, we conduct the simulations applying the linear pricing model.

The simulations are repetitively conducted for an offloading time period equal to $\Delta T=5 \mathrm{sec}$. The data volume needs of the UEs follow a uniform distribution of the file sizes between $5-$ $15 \mathrm{MB}$. These data needs represent the volume of a photo to a small video, created by contemporary smartphones. The uplink power level of $\mathrm{UE}_{i}$ 's LTE interface card, $P_{i}^{L T E}$, is assumed to follow (1). The 802.11 network interface card power levels $P_{T x}^{W i F i}, P_{R x}^{W i F i}, P_{i d l e}^{W i F i}$ and $P_{\text {sleep }}^{W i F i}$ are assumed to follow the 


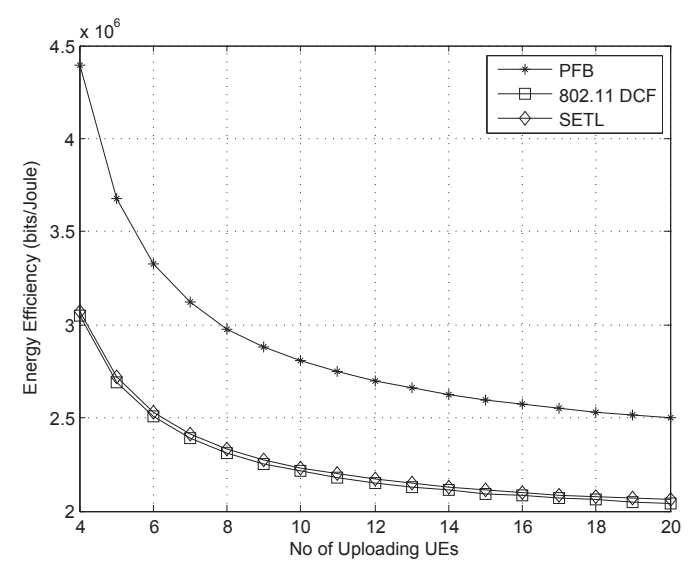

Fig. 4. Energy efficiency for $\theta_{i} \in[0.6,0.8]$ with linear pricing.

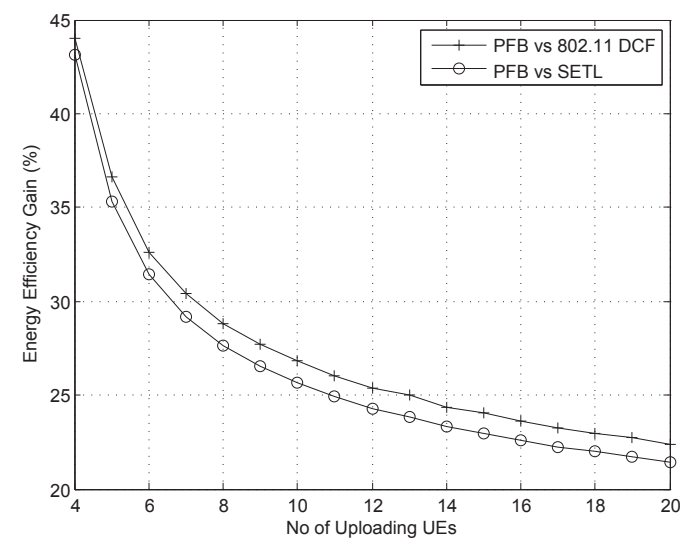

Fig. 5. Energy efficiency gain for $\theta_{i} \in[0.6,0.8]$ with linear pricing.

measurements provided in [16]. The numerical values of the simulation parameters are presented in Table I.

In Fig. 2 we present the energy efficiency of PFB, 802.11 and SETL, for different number of UEs ranging from four to 20 , with $\theta_{i} \in[0.8,1]$. The LTE uplink rate allocation follows the linear pricing model. In Fig. 3 the energy efficiency gain (\%) is presented, comparing PFB to the 802.11 standard and the SETL algorithm. In Fig. 4 we present the energy efficiency of PFB, 802.11 and SETL, for $\theta_{i} \in[0.6,0.8]$ while in Fig. 5 the energy efficiency gain (\%) is presented, comparing PFB to the 802.11 standard and the SETL algorithm.

\section{CONCLUSION}

In this paper we focused on the IFOM technique for uplink offloading that allows the concurrent transmission through WiFi and LTE. UEs are scheduled to upload through the WiFi following a proportionally fair allocation algorithm, where both the data load and the LTE spectrum efficiency is considered in the weighted fairness. Regarding the WiFi access scheme we presented our implementation considerations and proposed a scheme that provides for improved exploitation of the WiFi capabilities. For the LTE uplink rate allocation we proposed a linear pricing model for rate allocation. We presented energy efficiency simulation results, comparing the performance of the proportionally fair access with the standard 802.11 and the SETL algorithm.

\section{ACKNOWLEDGMENTS}

This work has been partially funded by the Research Projects GREENET (PITN-GA-2010-264759), AGAUR (2014-SGR-1551) and GEOCOM (TEC2011-27723-C02-01). This work was also co-funded by the Greek State Scholarship Foundation (IKY), following a procedure of individualised assessment of the academic year 2011-2012, through the resources of the educational program "Education and Lifelong Learning" of the European Social Fund (ESF) and the NSRF 2007-2013.

\section{REFERENCES}

[1] Cisco, "Cisco Visual Networking Index: Global Mobile Data Traffic Forecast Update, 2013-2018," Feb. 2014.

[2] U. Paul, A. P. Subramanian, M. M. Buddhikot, and S. R. Das, "Understanding Traffic Dynamics in Cellular Data Networks," in Proc. of IEEE INFOCOM 2011, Apr. 2011.

[3] J. Huang, F. Qian, A. Gerber, Z. M. Mao, S. Sen, and O. Spatscheck, "A Close Examination of performance and Power Characteristics of $4 \mathrm{G}$ LTE Networks," in Proc. of the 10th ACM International Conference on Mobile Systems, Applications, and Services, June 2012.

[4] C. Sankaran, "Data Offloading Techniques in 3GPP Rel-10 Networks: A tutorial," IEEE Communications Magazine, vol. 50, no. 6, pp. 46-53, June 2012

[5] ETSI, "3GPP TS 23.261: IP flow mobility and seamless Wireless Local Area Network (WLAN) offload; Stage 2 (v11.0.0)," Sep. 2012.

[6] H. Son, S. Lee, S.-C. Kim, and Y.-S. Shin, "Soft Load Balancing Over Heterogeneous Wireless Networks," IEEE Transactions on Vehicular Technology, vol. 57, no. 4, pp. 2632-2638, July 2008.

[7] V. Miliotis, L. Alonso, and C. Verikoukis, "Offloading With IFOM: The Uplink Case," in Proc. of IEEE International Conference on Communications (ICC), Dec. 2014.

[8] C. U. Saraydar, N. B. Mandayam, and D. Goodman, "Efficient Power Control via Pricing in Wireless Data Networks," IEEE Trans. on Communications, vol. 50, no. 2, pp. 291-303, 2002.

[9] P. Liu, P. Zhang, S. Jordan, and M. L. Honig, "Single-Cell Forward Link Power Allocation Using Pricing in Wireless Networks," IEEE Trans. on Wireless Communications, vol. 3, no. 2, pp. 533-543, 2004.

[10] "IEEE Standard for Information technology - Telecommunications and information exchange between systems - Local and metropolitan area networks - Specific requirements - Part 11: Wireless LAN Medium Access Control (MAC) and Physical Layer (PHY) specifications," IEEE Std 802.11-2007 (Revision of IEEE Std 802.11-2007), 2012.

[11] G. Bianchi, "Performance Analysis of the ieee 802.11 Distributed Coordination Function," IEEE JSAC, vol. 18, no. 3, pp. 535-547, Mar. 2000.

[12] F. P. Kelly, A. K. Maulloo, and D. K. Tan, "Rate control for communication networks: shadow prices, proportional fairness and stability," Journal of the Operational Research society, pp. 237-252, 1998.

[13] S. Boyd and L. Vandenberghe, Convex Optimization. Cambridge University Press, 2009.

[14] Y. Li, D. Papagiannaki, and A. Sheth, "Uplink Traffic Control in Home 802.11 Wireless Networks," in Proc. of the 2nd ACM SIGCOMM Workshop on Home Networks, Aug. 2011.

[15] C. H. Ke, C. C. Wei, K. W. Lin, and J. W. Ding, "A Smart ExponentialThreshold-Linear Backoff Mechanism for ieee 802.11 WLANs," International Journal of Communication Systems, vol. 24, no. 8, pp. 10331048, Aug. 2011.

[16] J. Ebert, S. Aier, G. Kofahl, A. Becker, B. Burns, and A. Wolisz, "Measurement and Simulation of the Energy Consumption of an WLAN Interface," Technical University of Berlin, Telecommunication Networks Group, Tech. Rep. TKN-02-010, June 2002. 